\title{
Utilização do TLM no Estudo do ADSL na Presença de Distúrbios Não Contínuos
}

\author{
Antonio Carlos Pinho ${ }^{1}$, Gianfranco Muncinelli ${ }^{1,2}$
}

\begin{abstract}
Resumo: O presente trabalho apresenta uma combinação do método das linhas de transmissão (TLM) com análise de Fourier buscando refinar o modelo do par trançado usado na transmissão ADSL. A contribuição do trabalho é a análise e utilização do DMT em termos de tensão e não de distribuição espectral de potência, permitindo um estudo do comportamento do sinal na presença da sinalização, que age como distúrbio.
\end{abstract}

Palavras-Chave: ADSL, DMT, sinalização de linha, par trançado.

Abstract: This paper presents the use of the transmission line method combinated with fourier analysis, aiming to refine the model of the twisted pair, in special way the use in ADSL transmission. The main goal is the analysis in terms of voltage - the general analysis most of times refers to PSD (power spectrum distribution) - that allows knowing the DMT bin in a very detailed way. The effects of impairments such ringing, can be studied.

Keywords - ADSL, DMT, ringing, twisted pair.

\section{INTRODUÇÃO}

A tecnologia ADSL (asymmetric digital subscriber line) tem demonstrado sua capacidade de ser uma ótima solução para acesso a redes de comunicação de dados e internet, onde um sinal de alta freqüência (se comparado com a freqüência de operação da rede telefônica) será transmitido pela rede telefônica, que é otimizada para sinais de menor freqüência (até $4 \mathrm{kHz}$ ) [1,2,].

O presente trabalho tem como objetivo contribuir com o modelamento do par trançado mediante a utilização do Método da Linha de Transmissão (doravante chamado TLM, abreviatura do inglês de Transmission Line Modelling) adequadas ao fenômeno da transmissão e reflexão de sinal $[3,4,5]$. A idéia principal é obter um modelo do par trançado que traga informações sobre a integridade do sinal transmitido via par trançado, na presença de distúrbios não contínuos (sinalização).

O método das linhas de transmissão (TLM) é bastante apropriado para estudos de interferência (EMI) e compatibilidade eletromagnética (EMC) [3]. Por ser um método diferencial no domínio do tempo, a análise de transientes pode ser feita com modelos de rápida aplicação.

acpinho@utfpr.edu.br; UTFPR/CPGEI/DAELT ${ }^{1}$, Av. 7 de Setembro, 3165 - 80230-901 - Curitiba - PR - Brasil1'. gianm@brasiltelecom.com.br; Brasil Telecom² Av. Madre Benvenuta, 2080 - 88035-900 - Florianópolis $\mathrm{SC}-\mathrm{Brasil}^{2}$
A contribuição maior é no tratamento do DMT em termos de tensão e não de potência como é costume na literatura. Este fato permite que os bins do DMT sejam conhecidos com mais profundidade e modelados individualmente. Sendo assim, pode-se obter informações específicas sobre faixas distintas de freqüências de operação do ADSL, isolando e analisando cada freqüência individualmente.

\section{ADSL}

ADSL é uma tecnologia de modem muito sofisticada, ou seja, uma tecnologia de codificação com encapsulamentos de protocolos de camadas mais altas.

A transmissão do ADSL é sensível ao comprimento da linha entre o assinante e a central telefônica onde estão os DSLAMs (digital subscriber line access multiplexer), sendo seu desempenho proporcional ao comprimento do loop, composto por cabos com fios de bitolas variadas $[1,5,6,7]$.

\section{CODIFICAÇÃO DO SINAL ADSL}

A abordagem da técnica de codificação se restringirá ao Discrete Multitone (DMT), pois é a técnica mais usada em aplicações comerciais $[1,8,9]$.

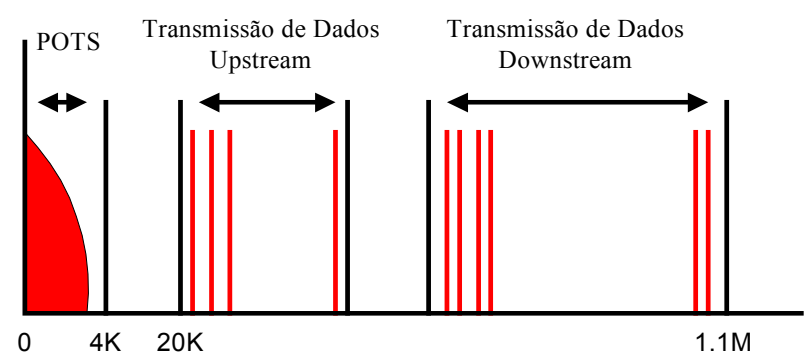

Fig. 1. Bandas do Sistema

A técnica DMT divide o espectro em 256 canais, de $4,3125 \mathrm{kHz}$ de largura cada, chamados "bins". Utiliza-se modulação de amplitude e fase para o transporte de dados em cada canal, que é monitorado individualmente na relação sinal ruído e, dependendo desta relação, será alimentado com mais ou menos bits [8].

$\mathrm{O}$ modem pode modular cada um destes canais com uma densidade de bit diferente (até um máximo de 15 bits $/$ segundo/ $/ \mathrm{Hz}$ ou $60 \mathrm{kbps} / 4 \mathrm{kHz}$ tom) dependendo do ruído da linha. Em baixas freqüências, onde existem menos interferências, a linha pode suportar $10 \mathrm{bits} / \mathrm{segundo} / \mathrm{Hz}$, 
enquanto que em altas freqüências este valor pode cair de 10 para 4 bits/segundo/Hz devido a um decréscimo correspondente de largura de banda. Este uso de subportadoras é bem mais complexo do que as solicitações de processamento do CAP; este fato torna DMT mais robusto que CAP [9].

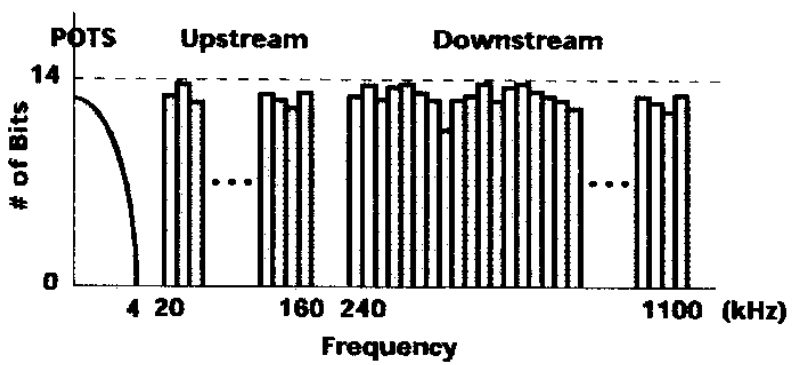

Fig. 2. Canais da Modulação DMT

A modulação DMT (Discrete Multi Tone) foi escolhida pelo American National Standards Institute (ANSI) como padrão pela norma T1.413 [10].

\section{MÉTODO TLM}

O TLM é um método diferencial utilizado para a modelagem no domínio do tempo (existe formulação para freqüência), sendo que o domínio de estudo é discretizado na forma de segmentos de linha de transmissão em uma dimensão, caracterizados por um conjunto de componentes $(R, L, C$ e $G$ ), em subdomínios onde são executados os cálculos. A figura 3 ilustra a linha de transmissão.

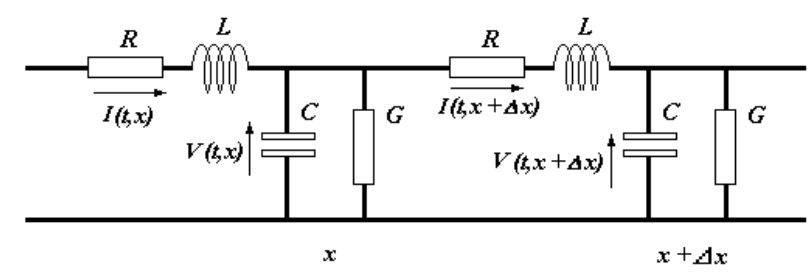

Fig. 3 - Linha de transmissão com parâmetros distribuídos.

O método numérico da modelagem por linhas de transmissão (TLM) é bastante apropriado para estudos de interferência (EMI) e compatibilidade eletromagnética (EMC) [3]. Por ser um método diferencial no domínio do tempo, a análise de transientes pode ser feita com modelos de rápida aplicação.

O TLM no domínio da freqüência encontra-se em estado de evolução bem menor do que no domínio do tempo. Neste caso a excitação é feita em um range de freqüências, obtendo-se respostas mais precisas para cada freqüência inserida. Admitindo que o objetivo é estudar o fenômeno de forma qualitativa, os resultados obtidos com TLM no domínio do tempo são aceitáveis.

\section{Modelagem}

Este item tem por objetivo abordar a modelagem que será utilizada nos cálculos e justificar algumas simplificações que serão adotadas.

\section{A. Modelagem do Par Trançado}

A estrutura do par metálico associada à rede de acesso ao sistema ADSL é extremamente complexa no que diz respeito a divisores (splitters); emendas; bitolas diferentes do fio; trechos primários, secundários e drop; rede interna do assinante e rede interna à central telefônica. $\mathrm{O}$ objetivo deste trabalho é estudar a influência de distúrbios não contínuos no sinal ADSL, portanto, baseados nas normas e em trabalhos referenciados, no modelo adotado todos os trechos de cabeamento (interno ao ATU-R e ATU-C, externo, primário, secundário, etc) serão representados por uma única bitola de fio.

Sabe-se que R, L, C e G são variáveis conforme a temperatura e que $\mathrm{R}$ e $\mathrm{L}$ são variáveis com a freqüência. Segundo a norma [13], R, G e L variam ainda conforme o revestimento (papel ou polietileno) (adotou-se polietileno como revestimento padrão para as simulações).

\section{B. Modelagem da Carga}

Um modelo apurado da carga deve levar em conta a impedância do divisor (splitter) e a impedância relacionada ao circuito equivalente formado pelo par trançado que leva ao aparelho telefônico, o modem e os pares trançados entre o divisor e o modem e entre o divisor e o aparelho telefônico. Simplificou-se o valor da carga para uma resistência pura de $100 \Omega$, porém o aplicativo gerado possui a capacidade de operar com outros valores de carga.

\section{Modelagem do Sinal ADSL}

A norma ANSI T1E1.413 [10] define as especificações do sinal ADSL, estabelecendo que a máxima transferência de densidade de potência da central telefônica sobre a linha telefônica é de $-40 \mathrm{dBm} / \mathrm{Hz}$. Com a banda de freqüência dos transmissores entre $25 \mathrm{kHz}$ e $1,1 \mathrm{MHz}$ (figura 2), significa que a potência máxima num link ADSL é de $20 \mathrm{dBm}$. Este número é citado com freqüência pela indústria e define o conteúdo de potência de um link ADSL [18], e é a máxima potência que pode ser enviada pelo DSLAM, lembrando que o ATU-R receberá bem menos potência, pois haverá a atenuação proporcionada pelo par trançado, incluindo-se o limite de transmissão ditado pela atenuação e distância. Em linhas mais curtas, haverá redução do envio de potência, pois durante a sincronização inicial haverá a troca destas informações entre ATU-C e ATU-R [18].

Em uma implementação DMT para ADSL, cada portadora tem sua própria taxa individual de modulação de dados. A taxa de modulação variável pode causar picos de tensão muito altos do sinal ADSL que são transmitidos pelo par trançado. Cada portadora (bin) possui uma freqüência e fase 
independentes, criando uma distribuição estatística de amplitude de sinal composta [18]. Poderá haver alinhamento das fases das múltiplas portadoras de maneira que haja um somatório dos picos de tensão individuais de modo que a forma de onda composta apresente uma amplitude de pico maior. A medida desta amplitude de pico em valor eficaz é chamada de Fator Crest (crest factor) que, para DMT ADSL é tipicamente 5,6 ou $15 \mathrm{~dB}$. Para este valor de fator crest, a probabilidade de haver tensões maiores é reduzida para aproximadamente 1 x 10-7.

\section{Valores de Tensão das Subportadoras do DMT-ATU-C}

A densidade espectral de potência é definida em $\mathrm{dBm} / \mathrm{Hz}$ [19]. No estudo atual, é interessante obter uma aproximação do sinal em termos de tensão. Além da PSD máxima, a norma (G.922.1) DMT prevê que a PSD nominal média dentro da banda de downstream não deve ultrapassar -40 $\mathrm{dBm} / \mathrm{Hz}$, que é o valor que ocorre durante a equalização inicial (training) onde ATU-C e ATU-R aplicam os ajustes de potência necessários para a transmissão.

Assim sendo, a potência nominal de downstream em um subcanal (tom ou bin) do DMT pode ser obtida [19] por:

$$
\begin{aligned}
& \mathrm{PD}=\text { PSD nominal } * 4,3125 \mathrm{kHz} \\
& \mathrm{PD}=-40 \mathrm{dBm} / \mathrm{Hz}+10 \log 4312,5 \\
& \mathrm{PD}=-3,6527 \mathrm{dBm} \\
& \mathrm{PD}=0,43125 \mathrm{~mW}
\end{aligned}
$$

Esse subcanal é referido como uma onda senoidal pura, então pode-se obter a amplitude da onda EFICAZ:

$$
\begin{aligned}
& \mathrm{Vef}=\sqrt{ }(\mathrm{PSD} * \mathrm{R}) \\
& \mathrm{Vef}=\sqrt{ }(0,43125 \mathrm{~mW} * 100 \Omega) \\
& \mathrm{Vef}=0,21 \mathrm{~V}
\end{aligned}
$$

Obtendo a tensão de pico aplicada aos condutores A e B (tip e ring):

$$
\begin{aligned}
& \text { Vpico }=\text { Vef } * 1,4142 \\
& \text { Vpico }=0,3 \mathrm{~V}
\end{aligned}
$$

Se todos os subcanais são utilizados e ele todos estão com o nível de PSD nominal, é possível calcular a potência de transmissão nominal agregada:

$$
\begin{aligned}
& \mathrm{PA}=\text { nominal PSD } *(1104 \mathrm{kHz}-25,875 \mathrm{kHz}) \\
& \mathrm{PA}=-40 \mathrm{dBm} / \mathrm{Hz}+10 \log 1078125 \\
& \mathrm{PA}=20,33 \mathrm{dBm} \\
& \mathrm{PA}=107,895 \mathrm{~mW}
\end{aligned}
$$

A tensão media de downstream de todo o sinal pode ser calculada então:

$$
\begin{aligned}
& \mathrm{Vef}=\sqrt{ }(\mathrm{PSD} * \mathrm{R}) \\
& \mathrm{Vef}=\sqrt{ }(107,895 \mathrm{~mW} * 100 \Omega) \\
& \mathrm{Vef}=3,285 \mathrm{~V}
\end{aligned}
$$

Lembrando que este não é mais um sinal senoidal puro; é possível definir o sinal DMT agora como sendo um sinal randômico de ruído branco, em função da mudança individual do subcanal de amplitude e fase ocorrerem simultaneamente. $\mathrm{Na}$ ocorrência de que a maioria dos subcanais atinjam juntos a sua respectivas amplitudes máximas de tensão, haverá a criação de um pico extrememente alto, cujo estudo estatístico define o fator crest, descrito anteriormente, e atinge 5,3 vezes o valor da amplitude. Assim sendo, é possível calcular tensão de pico, com até 10-7 BER (bit error rate), aplicada aos condutores A e B (tip e ring):

$$
\begin{aligned}
& \mathrm{V}=5,3 * 3,285 \\
& \mathrm{~V}=17,41 \text { Vpico ou } 34,82 \mathrm{~V} \text { pico a pico }
\end{aligned}
$$

\section{E. Valores de Tensão das Subportadoras do DMT-ATU-R}

De forma similar, calcula-se para as portadoras do ATU-R, apenas diferindo no valor de PSD máximo dentro da banda de downstream que é $-38 \mathrm{dBm} / \mathrm{Hz}$. Lembrando que o limite do DMT é $+3,5 \mathrm{~dB}$, a PSD máxima passa a ser $-38 \mathrm{dBm} / \mathrm{Hz}$ $+3,5 \mathrm{~dB}=-34,5 \mathrm{dBm} / \mathrm{Hz}$, obtemos:

$$
\text { Vpico }=0,37 \mathrm{~V}
$$

\section{F. Modelagem do Ringing}

Conhece-se por ringing o "toque do telefone" que é uma tensão $\mathrm{AC}$ superposta à uma tensão $\mathrm{DC}$, basicamente uma tensão não-sinusoidal com freqüência de $20 \mathrm{~Hz}$, $90 \mathrm{Vef}$ na fonte. Pode ocorrer variação em algumas centrais telefônicas com range de $162 / 3$ a $662 / 3 \mathrm{HZ}$ e tensões de 85 to $135 \mathrm{~V}$. Pela norma ANSI o limite máximo é $150 \mathrm{Vef}$, aplicado no condutor de "ring" (tip, ring, ground = A, B, terra).

O ringing é claramente um distúrbio não-contínuo. No início de cada burst do ringing há uma transição da tensão DC de $-48 \mathrm{~V}$ de alimentação para a superposição com a tensão AC. Os pulsos duram 2 segundos com a tensão com interrupções de 4 segundos. A representação da forma de onda do ringing é idealmente uma onda senoidal com seu eixo de simetria deslocado do zero em $-48 \mathrm{~V}$. O burst é caracterizado em centésimos de milisegundos, conforme mostrado na figura 4 [12].

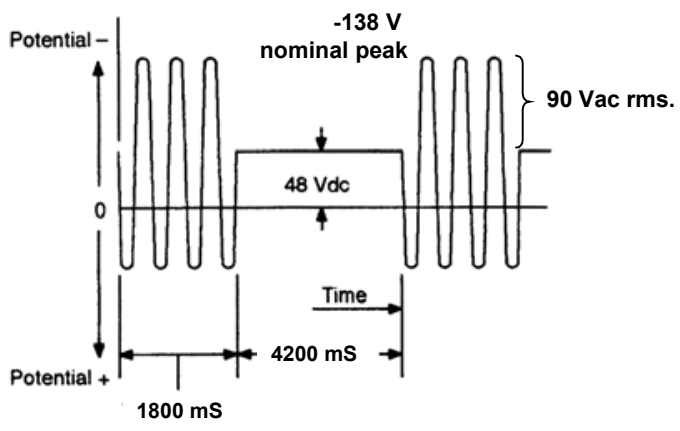

Figura 4. Potencial de ringing. 
O ringing não é balanceado, pois é aplicado em apenas um condutor (na literatura brasileira o condutor A e na literatura inglesa o condutor $\mathrm{R}$, de ringing). Esta aplicação não balanceada da tensão aumenta o acoplamento dentro do par trançado.

\section{ESTUDO DA INTERFERÊNCIA}

Tendo-se um sistema de transmissão e recepção composto pelo ATU-C e pelo ATU-R, (onde um transmite e o outro recebe, e vice-versa). O fluxo de sinal na direção do ATU-R para o ATU-C (transmissão do ATU-R para ATU-C) ocorre na faixa de upload $(20 \mathrm{kHz}$ a $160 \mathrm{kHz})$ citada no DMT. Já o fluxo de recepção do ATU-R (transmissão do ATU-C para ATU-R) ocorre na faixa de download $(160 \mathrm{kHz}$ a $1,1 \mathrm{MHz})$ do DMT.

O fluxo do distúrbio causado pelo ringing se dá no sentido do ATU-C para o ATU-R, pois é gerado pela central de comutação e será recebido pelo aparelho telefônico, localizado na mesma ponta que o ATU-R.

Para estudar a interferência causada pelo distúrbio não contínuo do ringing, serão feitas simulações com o aplicativo para os dois sistemas apresentados a seguir:

O sistema onde a excitação é o ringing e a carga é o ATU-R, mostrado na figura 5. A excitação é composta pela onda senoidal do ringing, com amplitude de $138 \mathrm{Vpico}$; freqüência de $20 \mathrm{~Hz}$, aplicada durante 1,8 segundos.

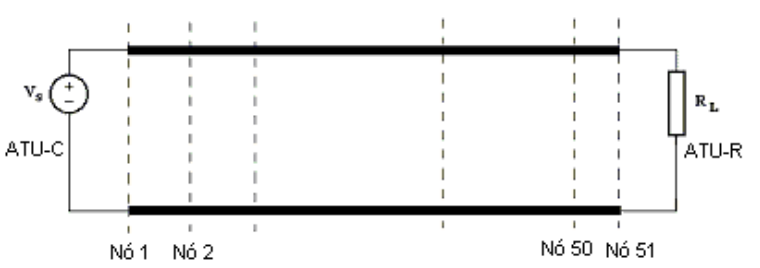

Figura 5. Sistema onde a excitação é o ringing e a carga é o ATU-R.

Na figura 6 é apresentado o sistema onde a excitação é uma subportadora do DMT e a carga é o ATU-C.

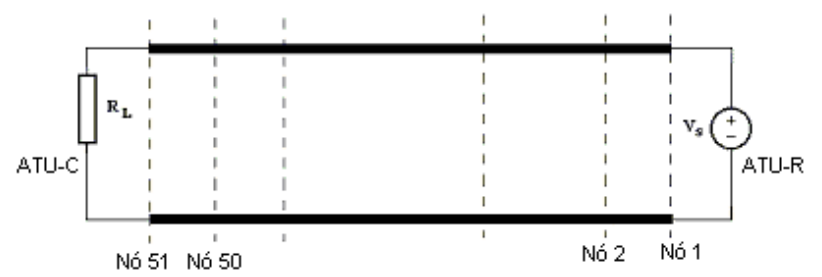

Figura 6. Sistema onde a excitação é subportadora do DMT e a carga é $O A T U-C$.

O nível de interferência máximo do ringing é nas proximidades da geração do mesmo (próximo ao ATU-C), que coincide com os pontos onde o sinal emitido pelo ATU$\mathrm{R}$ encontra-se em seus níveis mínimos. Dessa forma, as simulações serão realizadas analisando-se o nó 2 do sistema composto pelo ringing (segundo nó de maior nível de sinal) e o nó 50 (penúltimo nó, quase na carga do sistema onde a carga é representada pelo ATU-C (equivalente ao nó 2 do sistema anterior).

Para analisar a interferência do ringing na recepção do sinal do ATU-C, que recebe o sinal de upload do ATU-R nas baixas freqüências, serão analisadas algumas das freqüências da faixa de interesse. São elas: $20 \mathrm{kHz}, 40, \mathrm{kHz}$, $100 \mathrm{kHz}$ e $150 \mathrm{kHz}$, onda senoidal, com nível de sinal de $0,37 \mathrm{~V}_{\text {pico. }}$.

Foi usado o aplicativo TLM batizado de LTDTRING, desenvolvido inicialmente em [3] e alterado em [22] incluindo a capacidade de excitar o sistema utilizando o ringing.

\section{TRANSFORMADA DE FOURIER}

O sinal de ringing, bem como os sinais de interferência localizados, são representados no domínio do tempo. No Capítulo 4 foi visto que a modulação DMT é mais bem visualizada em freqüência. Sendo assim, para nossa análise, é necessário realizar uma análise em freqüência, para tanto, utilizaremos uma FFT (Fast Fourier Transform) [20].

Levando em conta que o ADSL usa a modulação DMT, é necessária uma Transformada de Fourier de, pelo menos, 1024 pontos com 4,416 MSPS (mega samples per second), que apresenta 512 células cada uma com largura de 4,31265 $\mathrm{kHz}$, e uma freqüência máxima de 2,208 MHz, suficiente para análise do sinal ADSL, que atinge máximo de 1,1 MHz.

Para uma maior portabilidade e facilidade no uso dos resultados, optou-se por utilizar o cálculo da FFT via uma planilha do MS Excel, fruto do trabalho do grupo Maxim Dallas Semiconductor [21]. Esta planilha fornece a FFT, bem como gráficos do sinal original e do sinal após a FFT.

\section{SiMULAÇÃO COM O RINGING}

O sinal de ringing foi descrito de forma completa em 5.F, porém, para efeito de modelagem, adota-se que, idealmente, é um sinal senoidal, com tensão de 90 Vef (138 Volts de pico), freqüência de $20 \mathrm{~Hz}$, aplicado durante 1,8 segundos e retirado durante os 4,2 segundos seguintes. O patamar DC associado de $-48 \mathrm{~V}$ não será modelado, pois os divisores (splitters) têm também a função de filtragem DC.

A carga será modelada por uma resistência pura de $100 \mathrm{~W}$. 
Tabela 1 Parâmetros elétricos e de simulação para o loop C \#6 em $20 \mathrm{~Hz}$ [13].

\begin{tabular}{|l|c|}
\hline CSA loop\#6 - fio 24AWG & Características (a 20Hz \\
\hline Comprimento da linha (metro) & 2744 \\
\hline Número de nós & 51 \\
\hline Número de iterações & 512 \\
\hline Resistência da linha por unidade de comprimento & $1,75 \mathrm{E}-01$ \\
\hline Condutância da linha por unidade de comprimento & 0 \\
\hline Capacitância da linha por unidade de comprimento & $5,00 \mathrm{E}-11$ \\
\hline Indutância da linha por unidade de comprimento & $7,03 \mathrm{E}-07$ \\
\hline Resistência da carga & 100 \\
\hline Resistência da fonte & 0 \\
\hline Indutância da carga & 0 \\
\hline
\end{tabular}

Gráfico da FFT

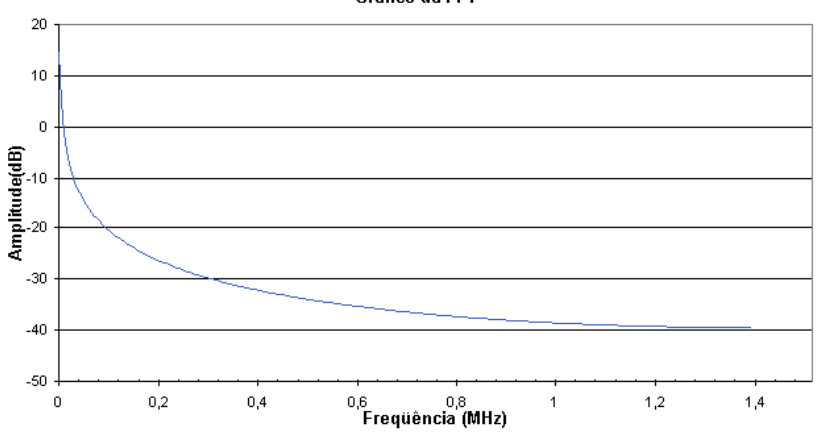

Figura 7. FFT do ringing calculada no nó 2, C loop \#6.

Os valores dos parâmetros elétricos dos fios do par trançado mudam em função da freqüência. No caso específico, para freqüência de $20 \mathrm{~Hz}$, a tabela 1 traz os valores dos parâmetros elétricos para o C loop\#6. Além das informações físicas, a tabela 1 mostra alguns parâmetros da simulação, como número de nós e número de passos de tempo (número de iterações) que são utilizados no LTDRING - que é o aplicativo modificado do original desenvolvido por Mauro Faccioni Filho e Golberi de Salvador Ferreira, publicado em [3]. As alterações realizadas, publicadas em [22], dizem respeito à inserção de novas excitações, contemplando o DMT e o ringing.

Usando o ringing como excitação, obtêm-se os seguintes resultados, calculados nos nós 2 e nó 51 (carga), apresentados nas figuras 7 e 8 respectivamente, após a FFT.

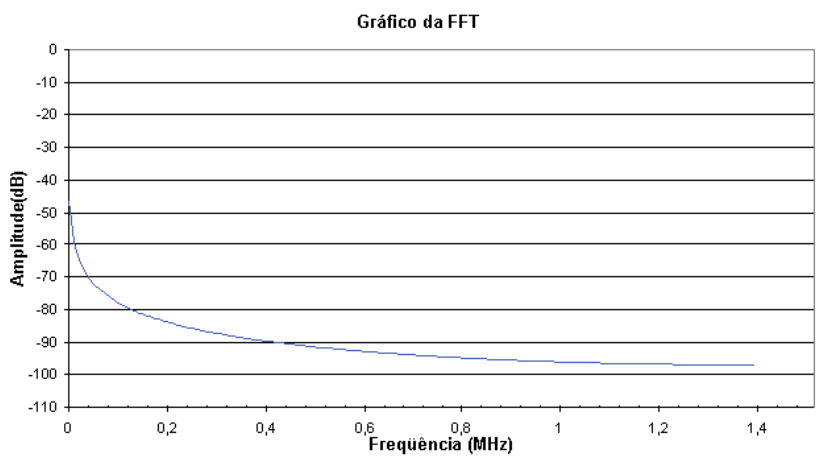

Figura 8. FFT do ringing calculada na carga (nó 51), C loop \#6.

Nota-se que o comportamento do fenômeno é semelhante, diferenciado apenas pela atenuação do sinal, em função da distância da fonte. Verifica-se que a influência da excitação é sentida exatamente no range de freqüências de operação do sinal do ADSL, conforme foi apresentado na figura 2.

\section{SIMULAÇÃO COM CANAIS DMT DO ADSL}

$\mathrm{Na}$ codificação DMT, o range de freqüências de operação está compreendido entre $20 \mathrm{kHz}$ e 1,1 MHz. O LTDTRING é utilizado para calcular a influência individual de cada bin levando em conta o valor de tensão associado e a freqüência de transmissão de bins específicos, visando a posterior comparação com o ringing.

Tabela 2. Parâmetros elétricos e de simulação para o loop CSA \#6 em $20 \mathrm{kHz}, 40 \mathrm{kHz}, 100 \mathrm{kHz}$ e $150 \mathrm{kHz}$ [13].

\begin{tabular}{|l|c|c|c|c|}
\hline C loop\#6 - fio 24AWG & $@ 20 \mathrm{kHz}$ & $@ 40 \mathrm{kHz}$ & $@ 100 \mathrm{kHz}$ & $@ 150 \mathrm{kHz}$ \\
\hline Comprimento (m) & 2744 & 2744 & 2744 & 2744 \\
\hline Número de nós & 51 & 51 & 51 & 51 \\
\hline Número de iterações & 512 & 512 & 512 & 512 \\
\hline Resistência / m & $1,76 \mathrm{E}-01$ & $1,80 \mathrm{E}-01$ & $2,00 \mathrm{E}-01$ & $2,23 \mathrm{E}-01$ \\
\hline Condutância / m & 0 & 0 & 0 & 0 \\
\hline Capacitância / m & $5,00 \mathrm{E}-11$ & $5,00 \mathrm{E}-11$ & $5,00 \mathrm{E}-11$ & $5,00 \mathrm{E}-11$ \\
\hline Indutância / m & $7,03 \mathrm{E}-07$ & $7,00 \mathrm{E}-07$ & $6,88 \mathrm{E}-07$ & $6,75 \mathrm{E}-07$ \\
\hline Resistência da carga & 100 & 100 & 100 & 100 \\
\hline Resistência da fonte & 0 & 0 & 0 & 0 \\
\hline Indutância da carga & 0 & 0 & 0 & 0 \\
\hline
\end{tabular}

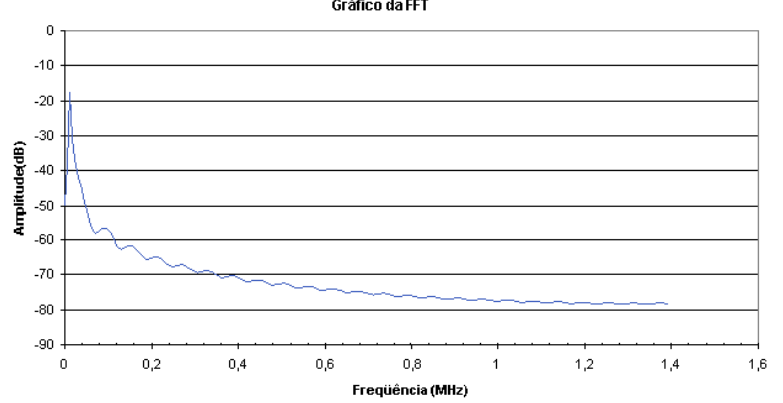

Figura 9. FFT do sinal em $20 \mathrm{kHz}$, calculado no nó 50, C loop \#6.

E assim sucessivamente para as freqüências de $40 \mathrm{kHz}, 100$ $\mathrm{kHz}$ e $150 \mathrm{kHz}$, cujas figuras não são mostradas por questão de espaço.

\section{SinAL X PERTURBAÇÃO}

O objetivo das simulações é identificar a influência da perturbação na transmissão do sinal do ADSL. A seguir são apresentadas as curvas sobrepostas do ringing e da 
subportadora em análise, visando a identificação desta influência. As simulações foram feitas para o C loop\#6, lembrando da equivalência entre os nós 2 e 50 dos dois sistemas mostrados nas figuras 5 e 6 . Ou seja, a sobreposição é de valores calculados no mesmo nó.

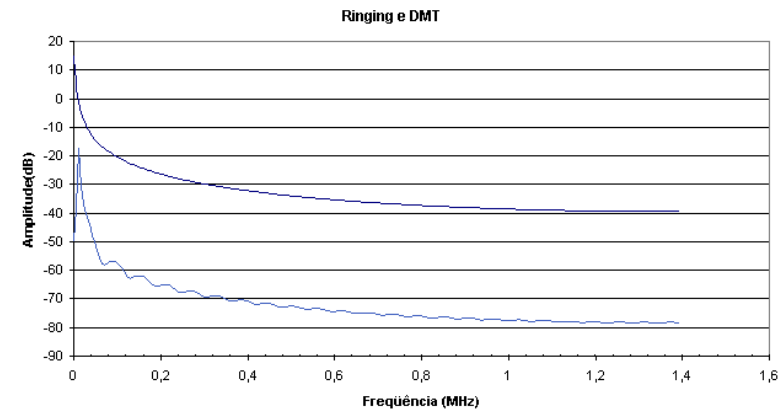

Figura 10. FFT do sinal em $20 \mathrm{kHz}$ e do ringing, C loop \#6.

Desta forma, procura-se comparar a resposta em freqüência das subportadoras de interesse com a resposta em freqüência do ringing. Na figura 10, há a superposição da figura 7 e da figura 9. A mesma superposição é aplicada para as outras freqüências de interesse e nota-se que a curva do sinal está sempre abaixo da curva do ringing. Estas superposições não são apresentadas por questão de espaço, mas podem ser encontradas em [22].

\section{CONCLUSÕES}

O TLM é um método numérico adequado ao estudo de fenômenos no domínio do tempo; usa-se uma excitação cuja forma de onda consiga excitar os modos de propagação no meio. A resposta é composta de dados no domínio do tempo, onde os diversos modos estão somados, ou seja, é um método onde as respostas transientes têm informações em diversas freqüências. A limitação é a excitação utilizada e a conversão para domínio da freqüência é feita pela aplicação da FFT.

Observando-se os resultados apresentados, nota-se que há influência dos distúrbios não contínuos simulados na banda utilizada para transmissão do ADSL. Esta influência implica na impossibilidade da recepção do sinal no momento do toque do telefone (ringing). Pelas curvas apresentadas é possível notar que o nível da perturbação excede o nível do sinal em praticamente todos as simulações realizadas, ocasionando uma completa indistinguibilidade do sinal. Em resumo, o sistema não funcionará neste intervalo de tempo. O ADSL, por meio do DMT, é adaptativo, e a conexão não cai em virtude do ringing, porém, pode haver renegociação inicial entre ATU-R e ATU-C e queda no throughput.

Apesar de não se haver encontrado na bibliografia a existência de testes e medições que comprovem o que foi afirmado acima, durante a operação dos equipamentos do sistema ADSL (o DSLAM especificamente) é possível notar que há uma interrupção da troca de células entre ATU-R e
ATU-C durante o momento do ringing. Ou seja, há uma observação prática da perturbação estudada.

\section{REFERÊNCIAS}

[1] GINSBURG, D.. Implementing ADSL. Massachusetts : Addison Wesley Longman Inc., 1999.

[2] MUNCINELLI, G.. Qualificação de Linha para Serviço ADSL. Anais do CININTEL 2001 - IV Congresso Internacional de Infraestrutura para Telecomunicações, 2001.

[3] FACCIONI FILHO, M.. O Método de Modelagem por Linhas de Transmissão (TLM) e Aplicações em Compatibilidade Eletromagnética (EMC). Dissertação de Mestrado. UFSC. 1997.

[4] CHRISTOPOULOS, C., Transmission-Line Modeling Method Short Course, Nottingham, UK, 1992.

[5] CHRISTOPOULOS, C., The Transmission-Line Modeling Method TLM, IEEE Press, New York, 1995.

[6] CORNET TECHNOLOGY INC. Digital Subscriber Line (DSL) Testing. Página da WEB, IEC - International Engineering Consortium, Web ProForum Tutorials, http://www.iec.org.

[7] BAKER, T.; PUCKETT, R.. Telephone Access Network Measurements Tektronix, 1998.

[8] LANE, J.. Personal Broadband Services: DSL and ATM. 1998, Virata USA.

[9] MUNCINELli, G.. ADSL - Aspectos de Codificação, Modulação e Correção de Erro. Anais do I2TS'2003 - International Information Technology Symposium. 2003.

[10] ANSI T1.413-1998. Network and Customer Installation Interfaces Asymmetric Digital Subscriber Line (ADSL) Metallic Interface. Norma do American National Standards Institute, 1998.

[11] GLOBESPAN. Spectral Compatibility of Digital Subscriber Line (DSL) Systems. Página da WEB, IEC - International Engineering Consortium, Web ProForum Tutorials, http://www.iec.org. Acesso em 28 de abril de 2006.

[12] BROWN, R.. Non-Continuous Events in the Telephone Outside Plant. TIA, 1999.

[13] ITU-T. Recommendation G-996.1 Test Procedures for Digital Subscriber Line (DSL) Transceivers. International Telecommunication Union - Telecomunication Standardization Sector, 1999.

[14] IKUMA, T.. Model-Based Identification of POTS Local Loops for DSL Connectivity Prediction. Dissertação de Mestrado. State University Virginia, 2001.

[15] WELSH D.D.. Investigation of Impedance and Mode Conversion of Telecommunications Cables for xDSL Systems Final Report (AY 3944). York EMC Services.

[16] WILKINSON, A.. ADSL/VDSL Line Simulation - A Feasibility Study and Initial Design. Dissertação de Mestrado. University College London, 1999.

[17] TIA-968-A. Technical Requirements for Connection of Terminal Equipment to the Telephone Network. Telecommunications Industry Association, 2002.

[18] QUARFOOT, J.. Managing ADSL Signals and Contending with Noise. CommsDesign.
Coms http://www.commsdesign.com/main/9812/9812feat1.htm. Acesso em 06/06/2006.

[19] WANG, R. Y.. ADSL Power Spectrum Density Calculation Application Report SPRA955. Texas Instruments. 2003. www.go-dsp.com. Acesso em 06/06/2006.

[20] LEE, E. A.; VARAIYA, P.. Structure and Interpretation of Signals and Systems. University of California, Berkeley, 2000.

[21] MAXIM DALLAS SEMICONDUCTOR. Crunching FFTs with Microsoft ${ }^{\circledR}$ Excel. APPLICATION NOTE 3292. http://www.maximic.com/appnotes.cfm/appnote_number/3292. Acesso em 02 de maio de 2006.

[22] MUNCINELLI, G.. Aplicação do Método das Linhas de Transmissão (TLM) no Estudo de Distúrbios Não Contínuos em Linhas Digitais Assimétricas de Assinante (ADSL). Dissertação de Mestrado. UTFPR Universidade Tecnológica Federal do Paraná, 2006. 